\title{
On the Construction of Energy-Efficient Broadcast Tree with Hitch-hiking in Wireless Networks
}

\author{
My T. Thai Yingshu Li Ding-Zhu Du
}

\author{
Department of Computer Science and Engineering \\ University of Minneapolis \\ Minneapolis, MN 55455 \\ \{mythai,yili,dzd\}@cs.umn.edu
}

\author{
Chunyu Ai \\ Department of Computer Science \\ HeilongJiang University \\ Harbin, China \\ aichuyu@hlju.edu.cn
}

\begin{abstract}
Due to the limited power supplies of a wireless node, energy efficiency is a crucial aspect to the design of a broadcast protocol. In the minimum energy broadcast problem, each node adjusts its transmission power to minimize the total energy consumption. The minimum energy broadcast problem is proved to be NP-Complete. The Hitch-hiking model introduced recently in [1] takes advantage of the physical layer to combine partial signals containing the same data in order to decode a complete message. Moreover, the Wireless Multicast Advantage (WMA), that is a single transmission can be received by all the nodes that are within the transmission range of a transmitting node, reduces the total energy of the broadcast tree. In this paper, we take advantages of both Hitch-hiking and WMA to design an energy-efficient Broadcast tree algorithm with Hitchhiking (BHH). The simulation results show that $\mathrm{BHH}$ reduces the total energy of the broadcast tree greatly.
\end{abstract}

Keywords: wireless networks, energy efficiency, broadcast algorithms, Hitch-hiking

\section{Introduction}

Recent improvements in affordable and efficient integrated electronic devices have a considerable impact on advancing the state of wireless networks in the form of ad hoc networks and sensor networks. Wireless networks are used in a wide range of applications including security, health care, military, and many other fields. However, a critical issue in wireless networks is power scarcity, driven in part by battery size and weight limitations. Therefore, energy efficiency is critical in designing any algorithm for wireless networks to prolong the network lifetime.

Many different algorithms for power management have been proposed recently. Power saving techniques can generally be classified into two categories: power saving by scheduling the wireless nodes to alternate between active and sleep states [12], [13]; and power control by adjusting the transmission range of each wireless node [14], [11].

In this paper, we study the problem of minimizing the total broadcast energy in wireless networks. The broadcast problem in wireless networks is to decide a transmission power level for each node so that the source node can broadcast to all the other nodes either directly or indirectly through the relay nodes with the objective of minimizing the total energy consumption. Our focus is on source-initiated broadcast. Our objective is to construct a minimum-power broadcast tree, rooted at the source node, including all the nodes. Our key idea is to reduce the total energy consumption of the broadcast tree by taking the Hitchhiking model [1] and Wireless Multicast Advantage (WMA) into consideration. By successfully combining partial signals to obtain complete information, we can efficiently reduce the total energy consumed in broadcasting data. Moreover, we exploit further the characteristics of WMA, that is to add a set of nodes at a time rather than just adding one node as in [2]. Without using Hitch-hiking model, the total power of our broadcast tree is $49 \%$ less than that of Broadcast Increment Power (BIP), which is a well-known algorithm for minimizing the total energy consumption of a broadcast tree.

The rest of this paper is organized as follows. In the next section, we present the communication model, the Hitch-hiking model, and the network model. Section 3 describes the related work. Next, in section 4 , we introduce our algorithm. The simulation results are presented in section 5. Finally, section 6 concludes our paper. 


\section{Preliminaries}

\subsection{Communication Model}

We assume that any node in the network can be used as a relay node to forward data to other nodes in the network. All nodes are equipped with omnidirectional antennas so that if node $i$ transmits data to node $j$, all nodes closer to $i$ than $j$ will also receive the transmitted data. We also assume that all nodes can adjust their power levels. Each node can choose its transmission power from 0 to some maximum value $p_{\max }$.

Attenuation model commonly assumes that signal power falls proportional to $d^{\alpha}$, where $d$ is the signal travelling distance, and $\alpha$ is an environmentally dependent real constant between 2 and 4 [6]. A node $j$ can properly receive a signal from a node $i$ which has a power level $p_{i}$ if $p_{i} \geq \gamma d_{i j}^{\alpha}$, where $\gamma$ represents the receiver's power threshold for signal detection or Signal to Noise Ratio (SNR), often normalized to 1. Hence, for node $i$ in the network, the power required to successfully transmit data to node $j$ is given by: $p_{i j} \geq d_{i j}^{\alpha}$.

\section{$2.2 \quad$ Hitch-hiking Model}

The Hitch-hiking model is first proposed in [1]. The key idea of the Hitch-hiking is to take advantage of not only full reception but also partial reception of signals. In [1], the authors introduced two thresholds on the SNR:

- $\gamma_{p}$ : threshold needed for successfully decoding the packet payload

- $\gamma_{a c q}$ : the threshold required for a successful time acquisition

where $\gamma_{a c q}<\gamma_{p}$

A packet received with a $\operatorname{SNR} \gamma$ is:

1. Full reception if $\gamma_{a c q}<\gamma_{p} \leq \gamma$

2. Partial reception if $\gamma_{a c q} \leq \gamma<\gamma_{p}$

3. Failed reception if $\gamma<\gamma_{a c q}$

The basic idea of the Hitch-hiking is that if a node receives the packet containing the same information $n$ times from different neighbors with $\gamma_{i}$ such as $\sum_{i=1}^{n} \gamma_{i} \geq \gamma_{p}$ and $\gamma_{i} \geq \gamma_{a c q}$ for all $i$, then that node can successfully receive the packet.

In designing the solution, the authors assume that $\gamma_{p}=1$ and $\gamma_{a c q}=0$ for simplicity.

\subsection{Network Model}

Let $V$ denote the collection of wireless nodes and let $G=(V, E)$ denote the directed graph on $V$ that contains all edges when each node transmits at its maximum transmission power. Every node $i \in V$ has an associated transmission power level $p_{i}$. When node $i$ transmits a packet, the amount of reception by node $j$ is quantified by the coverage of node $j$ defined as $C\left(\frac{\gamma_{i j}}{\gamma_{p}}\right)$ where $C(\beta)$ is the coverage function given by:

$$
C(\beta)= \begin{cases}1 & \text { if } \beta>1 \\ \beta & \text { if } \frac{\gamma_{a c q}}{\gamma_{p}}<\beta<1 \\ 0 & \text { otherwise }\end{cases}
$$

Thus, the coverage provided by node $i$ on node $j$ is:

$$
C(i j)= \begin{cases}1 & \text { if } \frac{p_{i}}{d_{i j}^{\alpha}} \geq \gamma_{p} \\ \frac{p_{i}}{d_{i j}^{\alpha}} & \text { if } \gamma_{a c q} \leq \frac{p_{i}}{d_{i j}^{\alpha}}<\gamma_{p} \\ 0 & \text { otherwise }\end{cases}
$$

Let a directed graph $T=\left(V, E^{\prime}\right)$ represent the broadcast tree where $V$ is the set of all the nodes in $G$ and $E^{\prime}$ is the set of all the edges corresponding to the communication links between nodes after the power level is set. For each directed edge $i j \in E^{\prime}$, the coverage provided by an edge $i j$ to the node $j$ is calculated by (1), excluding the 0 case since in order for an directed edge $i j$ to exist, the coverage must be greater than 0 .

\section{Related Work}

The problem of minimum-energy broadcast in wireless networks has received significant attention over the last few years. Liang [4] proves that this problem is NP-Complete. For an extensive discussion of minimum-energy broadcast tree algorithms, please refer to [5]. In this section, we briefly review some of previous work.

In [7], the authors design a localized protocol where each node only needs to know the knowledge of its distance to all its neighbors and distances between its paired neighbors. Some other research works that consider the broadcast tree construction in wireless networks are [8] and [9]. The authors in [8] proposed a globalized algorithm which builds a 2-node-connected graph and assume an arbitrary energy model. Three different integer programming models are illustrated in [9] to provide an optimal solution of the minimum power broadcast/multicast problem in wireless networks. Some near-optimal heuristics are given in [2], [10]. The proposed BLU algorithm first establishes a minimum-cost path from the source to each destination, and the broadcast tree is formed by the su- 
perposition of these unicast paths. However, the aggregation of these individual shortest paths does not guarantee that the resultant broadcast tree has the minimum total energy.

The works most relevant to our approach are [2] and [1]. Broadcast Increment Power (BIP) [2] is a popular heuristic to construct a minimum-energy broadcast tree rooted at the source node. BIP is similar to Prim's algorithm. Starting at the source node, BIP constructs the tree by first determining the node that the source can reach with the minimum incrementing of power at the source node. BIP continues by adding one node at a time to the tree. Each added node is an uncovered node which can be added to the tree at the minimum additional cost. The approximation ratio of BIP is between $\frac{13}{3}$ and 12 [3]. Note that BIP just adds one node at a time, thus failing to fully exploit the advantage of WMA.

Recently, the Hitch-hiking model, [1], which is to combine the partial signals to a completed signal has been introduced. In [1], the authors presented the Wireless Multicast with Hitch-hiking (WMH) algorithm. This algorithm has two phases. First, it constructs the Minimum Spanning Tree (MST) rooted at the source node. Starting from the source node, at each step, the algorithm chooses a fully covered node $u$ and sets the power level of node $u$ to the power needed to reach its furthest children and grandchildren in the MST while obtaining the maximum power reduction in $u$ 's local region. Once the power level of node $u$ is decided, $u$ 's power level will not be modified again. Note that WMH constructs the broadcast tree based on the MST and adjusts the power level of each node based on local optimization. It does not take full advantages of WMA and Hitch-hiking.

In the following section, we describe our algorithm that at each step, the algorithm adds a set of nodes into the tree to further exploit the properties of WMA. It also considers the partial signal that a node can obtain from other nodes, not only from its parent and grandparent.

\section{The Broadcast with Hitch-hiking Al- gorithm}

Let us first introduce the following notations:

- $c(j i)$ : the coverage of node $i$ provided by node $j$. $c(j i)$ is defined in formula (1)

- $c(i)$ : the total coverage of node $i$ provided by all the other nodes. $c(i)=\sum_{j \in V} c(j i)$

- $p_{h}(i)$ : the power level of node $i$ at the iteration $h$

- $N(i)$ : the neighbors of node $i$
Let $P_{h}$ be the set of nodes that are not fully covered in the network at the iteration $h$. Thus, $F_{h}=V-P_{h}$ is the set of nodes that are fully covered in the network at the iteration $h$. In the algorithm, sometimes we refer the set of fully covered nodes and the set of partially covered nodes as $F$ and $P$ respectively if we do not specify the iteration step. Let us define a ratio $r(i)$ :

$$
r(i)=\frac{p_{h}(i)-p_{h-1}(i)}{\sum_{j \in P_{h-1}} c(j)}
$$

Note that $p_{h}(i)-p_{h-1}(i)$ is the incremental power of node $i$, and $\sum_{i \in P_{h}} c(i)$ is the sum of the updated coverage of all nodes $j$ after increasing the power level of node $i$ where $j$ is in the partially covered node set of the previous iteration, $P_{h-1}$. To better illustrate this idea, please look at Figure 1.

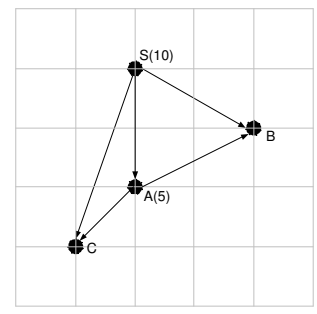

(a) Original graph $G$

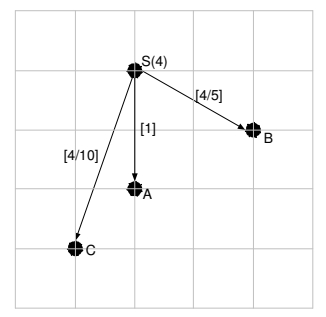

(b) Broadcast Tree $T$ at $h$ iteration

\section{Figure 1: An example for calculating $r$}

Figure 1(a) represents the network where the maximum power level of node $S$ and $A$ are 10 and 5 respectively. Figure 1(b) shows the broadcast tree $T$ at the $h$ iteration. At this step, $P_{h}=\{B, C\}$ and $F_{h}=\{S, A\}$. The ratio $r$ of node $S$ when increasing the power level of node $S$ to fully cover node $B$ is:

$$
r^{B}(S)=\frac{5-4}{1+\frac{5}{10}}=\frac{2}{3}=0.67
$$

And the ratio $r$ of node $A$ when increasing the power level of node $A$ to fully cover node $C$ is:

$$
r^{C}(A)=\frac{\frac{12}{10}}{\left(\frac{4}{10}+\frac{6}{10}\right)+1}=\frac{6}{10}=0.6
$$

In order to minimize the total power consumption, we not only want the incremental power of a node at each step to be small, but also want the sum of the coverage of all nodes to be large. This leads to the value $r(i)$ must be the minimum one at each iteration. Now, we are ready to introduce BHH.

Algorithm Description:

The BHH algorithm starts at the source node $S$. 
The initial coverage of $S$ is $c(S)=1$ and the initial coverage $c(i)=0$ for all $i \in V$ and $i \neq S$. Therefore, $F_{0}=\{S\}$ and $P_{0}=V-F_{0}$. Let $T=(V, \emptyset)$ be the initial broadcast tree. First, adjust the power level of node $S, p(S)$ to cover each node $i$ where $i \in N(S)$ and $i \in P_{0}$. At each power level, the ratio $r(S)$ is calculated by formula (1). The power level of node $S$ is set to the level at which $r(S)$ is the minimum one. We then update the coverage $c(k)$ where $k \in P_{0}$ and $k$ can fully or partially receive a signal from node $S$, and add all the directed edges from node $S$ to other nodes $k$ with the associated coverage covered to $T$. If $c(k) \geq 1$, remove $k$ from the partially covered node set $P$ and add it to the fully covered node set $F$. For all the nodes in $F$, we iteratively repeat the process until $P=\emptyset$, i.e, all the nodes in $T$ are fully covered. The algorithm is shown in Algorithm 1.

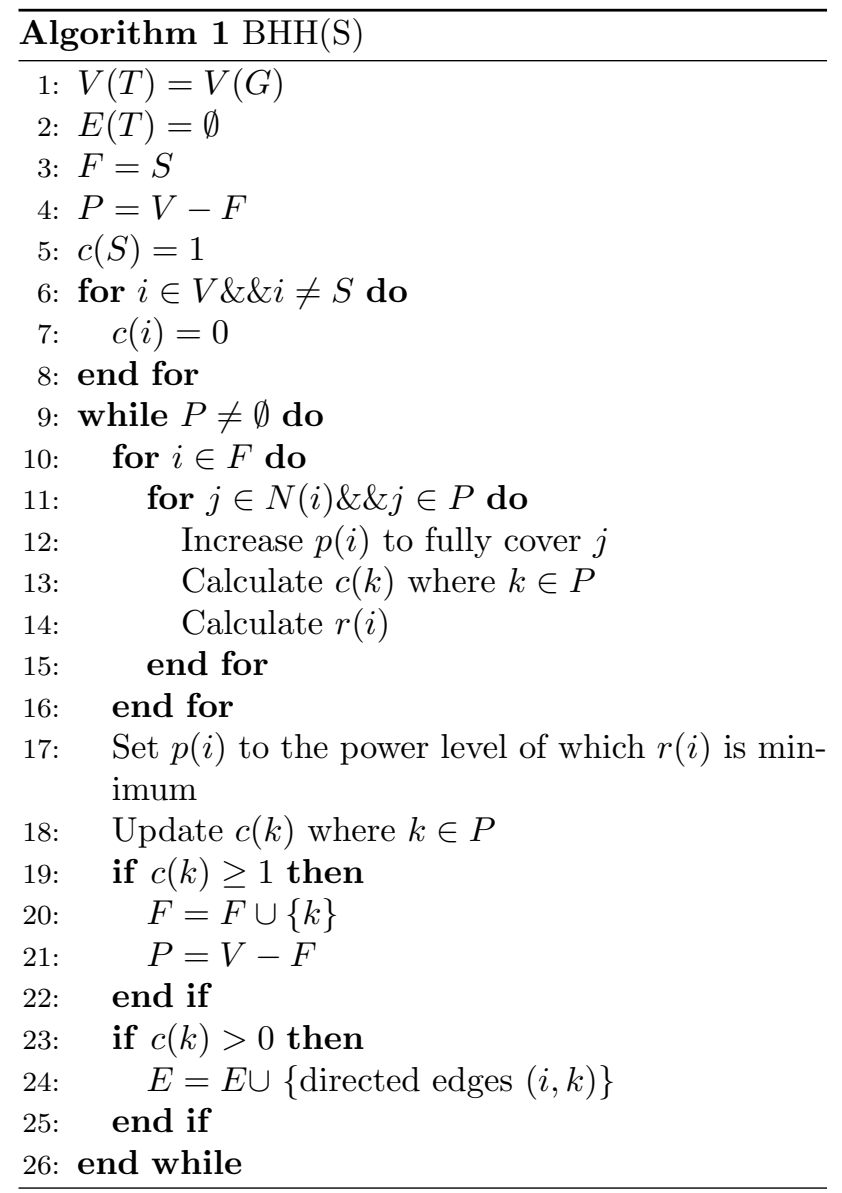

\section{Simulation Results}

In this section, we evaluate the performance of $\mathrm{BHH}$ by comparing it to another two algorithms, BIP and WMH. Since BIP does not use Hitch-hiking model, it is also our interest to compare our algorithm without

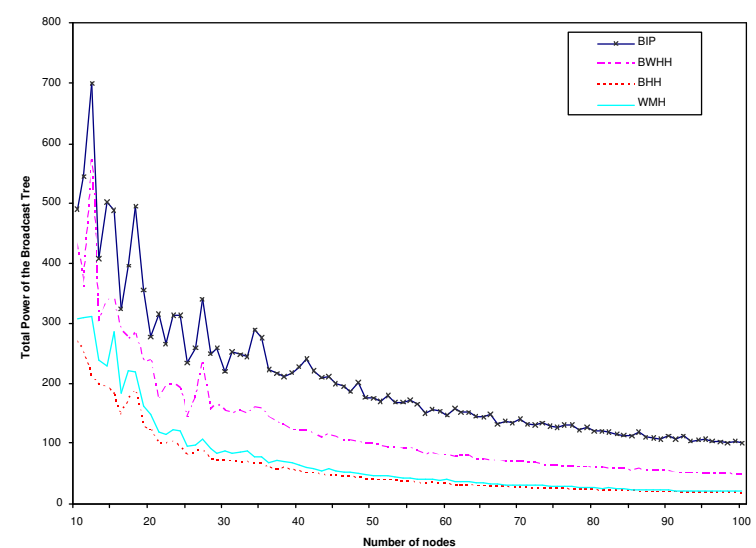

Figure 2: A comparison of total power, $\alpha=2$

using Hitch-hiking (BWHH) to BIP. The BWHH algorithm can be obtained from $\mathrm{BHH}$ with a change on the coverage function. Instead of using the formula (1), the coverage provided by node $i$ on node $j$ is defined as:

$$
C(i j)= \begin{cases}1 & \text { if } \frac{p_{i}}{d_{i j}^{\alpha}} \geq \gamma_{p} \\ 0 & \text { otherwise }\end{cases}
$$

In this simulation, we considered the following parameters:

- $n$ : the number of nodes in a network. We simulate a stationary network randomly distributed over the same deployment region, thereby increasing the network density when the number of nodes increases. $n$ is from 10 to 100 .

- $p_{\max }$ : The maximum power level of each node is randomly assigned on each simulation setup.

We ran 100 simulations for each value of $n$. In all simulations, a fixed value $\alpha=2$ was used. The performance metric is the average of the total power of the broadcast tree obtained from each algorithm.

Figure 2 illustrates the performance of the proposed algorithm $\mathrm{BHH}$ comparing to BIP and WMH. As can be seen, the total power of the broadcast tree constructed using $\mathrm{BHH}$ is almost $77 \%$ less than that of $\mathrm{BIP}$, and $15 \%$ less than that of WMH. Also, the total power of a broadcast tree decreases when the number of nodes increase. This is because as the network density increases, more nodes are available to work as relay nodes and the nodes are becoming closer.

Figure 2 also shows that the power of the broadcast tree constructed using BWHH is $49 \%$ less than that 


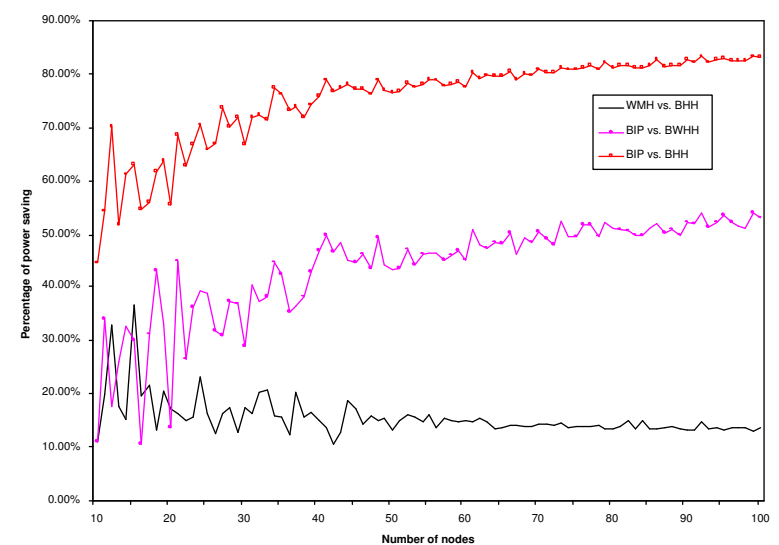

Figure 3: The improvement of $\mathrm{BHH}$

of BIP. This indicates that our algorithm takes more advantages of WMA than BIP does.

In Figure 3, we present the comparison of the improvement on saving energy over another three algorithms in percentage: BIP vs. BHH, BIP vs. BWHH, $\mathrm{BIP}$ vs. WMH. As can be seen in this figure, $\mathrm{BHH}$ is the one that can save the most energy, comparing to both BIP and WMH. This analysis indicates that combining WMA and Hitch-hiking model does achieve a better result.

\section{Conclusion}

In this paper, we studied the minimum-energy broadcast problem, which is proved to be NPComplete. We proposed the BHH algorithm based on the Hitch-hiking model. This algorithm takes advantages of WMA and of the Hitch-hiking concept. The simulation results showed that $\mathrm{BHH}$ can reduce the total power of the constructed broadcast tree by as much as $77 \%$ compared to BIP, and $15 \%$ compared to WMH. We also compared our algorithm without using Hitch-hiking to BIP, the total power of the broadcast tree can be reduced by $49 \%$.

It is our interest to further develop the distributed version of BHH in mobile environment where node mobility is highly concerned. We would also like to investigate the reliability and collision of the Hitch-hiking model.

\section{References}

[1] M. Agarwal, J. H. Cho, L. Gao, and J. Wu, "Energy Efficient Broadcast in Wireless Ad hoc Networks with Hitch-hiking," In Proc. of IEEE INFOCOM, 2004
[2] J. E. Weiselthier, G. D. Nguyen, and A. Ephremides, "On the Construction of Energy-Efficient Broadcast and Multicast Trees in Wireless Networks," IEEE INFOCOM, 2001

[3] P.-J. Wan, G. Calinescu, X.-Y., Li, and O. Frieder, "Minimum-energy broadcast routing in static ad hoc wireless networks," In Proc. of IEEE INFOCOM, 2001

[4] W. Liang, "Constructing Minimum-Energy Broadcast Trees In Wireless Ad Hoc Networks," In Proc. of MOBIHOC, 2002

[5] F. Ingelrest, D. Simplot-Ryl, and I. Stojmenovic, "Energy-Efficient Broadcasting in Wireless Mobile Ad Hoc Networks," in M. Cardei, I. Cardei, D.-Z. Du (eds.) Resource Management in Wireless Networking, Kluwer Academic Publishers, to appear in 2004

[6] T.S. Rappaport, "Wireless communications: principles and practice," Prentice Hall, 1996

[7] J. Cartigny, D. Simplot and I. Stojmenovic, "Localized minimum-energy broadcasting in ad-hoc networks," In Proceedings of IEEE INFOCOM, pp. 2210-2217, 2003.

[8] E. Lloyd, R. Liu, M. Marathe, R. Ramanathan, and S. Ravi, "Algorithmic aspects of topology control problems for ad hoc networks", In Proc. of Annual Workshop on Mobile and Ad Hoc Networking and Computing (MobiHoc), Lausanne, Switzerland, 2002.

[9] A. K. Das, R. J. Marks, M. El-Sharkawi, P. Arabshahi and A. Gray, "Minimum Power Broadcast Trees for Wireless Networks: Integer Programming Formulations",In Proceedings of IEEE INFOCOM, pp. 22102217, 2003.

[10] J. E. Wieselthier, G. D. Nguyen and A. Ephremides, "Algorithms for energy-efficient multicasting in static ad hoc wireless netoworks" Mobile Networks and Applications (MONET)6, pp. 251-263, 2001.

[11] J.E. Wieselthier, G. D. Nguyen, and A. Ephremides, "On constructing minimum spanning trees in $\mathrm{k}$ dimensional spaces and related problems," In Proc. of IEEE INFOCOM, 2000

[12] B. Chen, K. Jamieson, H. Balakrishman, and R. Morris, "SPAN: an energy-efficient coordination algorithm for topology maintenance in ad hoc wireless networks," ACM Wireless Netowrks Journal 2002, 481-494.

[13] S. Singh, C. Raghavendra, and J. Stepanek, "Poweraware broadcasting in mobile ad hoc networks," In IEEE PIMRC, 1999.

[14] R. Ramanthan and R. Hain, "Topology control of multihop wireless networks using transmit power adjustment," In Proc. of IEEE INFOCOM, 2000. 